\title{
Circular Dichroism in Atomic Resonance-Enhanced Few-Photon Ionization
}

\author{
A.H.N.C. De Silva, ${ }^{1}$ T. Moon, ${ }^{2}$ K.L. Romans,${ }^{1}$ B.P. Acharya,${ }^{1}$ S. Dubey,${ }^{1}$ K. \\ Foster,${ }^{1}$ O. Russ, ${ }^{1}$ C. Rischbieter, ${ }^{1}$ N. Douguet,${ }^{2}$ K. Bartschat,${ }^{3}$ and D. Fischer ${ }^{1}$ \\ ${ }^{1}$ Physics Department and LAMOR, Missouri University of Science 83 Technology, Rolla, MO 65409, USA \\ ${ }^{2}$ Department of Physics, Kennesaw State University, Kennesaw, Georgia 30144, USA \\ ${ }^{3}$ Department of Physics and Astronomy, Drake University, Des Moines, Iowa 50311, USA
}

(Dated: May 13, 2021)

\begin{abstract}
We investigate few-photon ionization of lithium atoms prepared in the polarized $2 p\left(m_{\ell}=+1\right)$ state when subjected to femtosecond light pulses with left- or right-handed circular polarization at wavelengths between $665 \mathrm{~nm}$ and $920 \mathrm{~nm}$. We consider whether ionization proceeds more favorably for the electric field co- or counter-rotating with the initial electronic current density. Strong asymmetries are found and quantitatively analyzed in terms of "circular dichroism" $(C D)$. While the intensity dependence of the measured $C D$ values is rather weak throughout the investigated regime, a very strong sensitivity on the center wavelength of the incoming radiation is observed. While the co-rotating situation overall prevails, the counter-rotating geometry is strongly favored around $800 \mathrm{~nm}$ due to the $2 p-3 s$ resonant transition, which can only be driven by counter-rotating fields. The observed features provide insights into the helicity dependence of light-atom interactions, and on the possible control of electron emission in atomic few-photon ionization by polarization-selective resonance enhancement.
\end{abstract}

\section{INTRODUCTION}

The response of matter to circularly polarized light can depend on the helicity of the light's polarization. This phenomenon is referred to as circular dichroism $(C D)$ and is widely exploited, e.g., in the analysis of chiral molecules (see Ref. [1] for an overview). Biomolecules, such as sugar or amino acids, are prominent examples of chiral targets for which single- [2-4] and multi-photon ionization [5-7], as well as tunnel ionization in intense laser fields [8], exhibit a dependence on the light's helicity even for randomly oriented molecules. These dichroic differences have even been suggested to be the symmetrybreaking cause of life's homochirality [9, 10]. Nevertheless, these effects are typically weak and vanish completely in the electric dipole approximation after integration over all possible target orientations.

For spatially aligned systems, in contrast, a $C D$ signal occurs already for electric dipole transitions, resulting in much stronger asymmetries [11]. The most fundamental realizations of such oriented targets are polarized atoms with a magnetic quantum number $m \neq 0$. In this situation, the helicity dependence is a result of either equal or opposite rotations of the electric field with respect to the initial current density of the active electron. Due to their fundamental importance, these benchmark systems have recently attracted considerable interest [12-19] in the study of the interaction between handed light and handed matter.

Atomic circular dichroism is quantitatively defined as

$$
C D=\frac{P_{+}-P_{-}}{P_{+}+P_{-}},
$$

where $P_{+}$and $P_{-}$are the cross sections for the co- and counter-rotating light polarizations respectively. In previous studies, it was found that there is no unambiguous answer to the fundamental question whether ionization proceeds more likely for the co- or the counter-rotating case. In the simplest case of one-photon ionization by a weak field, this question was already answered by Bethe and Salpeter, whose theoretical framework [20] delivers a $C D$ value of close to 1 , meaning that the co-rotating geometry is strongly favored $[12,21]$. To the contrary, in the nonadiabatic tunneling regime, i.e., for intense fields, the ionization cross section was found to be larger for a counter-rotating field, thus leading to negative $C D$ values.

In multi-photon ionization, the sign of the $C D$ value features a dependence on the intensity of the ionizing field $[17,22]$. This intensity dependence can be rather strong [18] due to "Freeman" resonances [23], where transient resonance enhancement occurs due to AC Stark shifts of intermediate levels.

In a recent Letter [19], two-photon ionization of polarized atoms was investigated in the case where resonance enhancement did not significantly affect the ionization rates. Lithium atoms were prepared in the $2 p(|m|=1)$ state and then ionized by a field $(671 \mathrm{~nm})$ tuned to the $2 s-2 p$ resonance transition. A positive and weakly intensity-dependent value of the CD was found throughout the investigated intensity regime, while strong helicity-selective Autler-Townes shifts were observed for the co-rotating geometry due to the coupling of the excited initial target state with its ground state. This peculiar feature allowed us to control not only the ionization probability, but also the photoelectron energy via the field polarization and intensity.

In the present study, we report an extension of our earlier work [19], now altering not only the intensity, but also the wavelength of the ionizing field. Specifically, we scan a wavelength range around the $2 p-3 s$ transition (at about $812 \mathrm{~nm}$ ). Close to this resonance, the $2 p$ state is ionized by the absorption of three photons, and resonance enhancement through the $3 s$ state, as well as Autler-Townes 
shifts due to the $2 p-3 s$ coupling, are expected to occur only for the counter-rotating geometry. In combination with the results reported in [19], the present work aims to understand the role of resonance enhancement in circular dichroism and the sensitivity of dichroic asymmetries to wavelength change of the ionizing radiation.

The experimental results are compared with theoretical predictions obtained by solving the time-dependent Schrödinger equation (TDSE). These results exhibit overall good agreement with the measured $C D$ values. As in our earlier study, the present system represents a fundamental realization of a chiral system and, therefore, provides fundamental insights into implications of asymmetries in light-matter interaction.

\section{EXPERIMENTAL SETUP}

Our experiment was performed with the same apparatus described in [19]. It consists of three components: An all-optical lithium atom trap (AOT) [24], a tunable femtosecond light source, and a reaction microscope that allows us to measure the electron and recoil ion momenta after an ionization process [25, 26].

In the AOT, lithium atoms are laser-cooled to a temperature of about $1 \mathrm{mK}$ in the field of continuous-wave laser beams near the $2 s-2 p$ resonance at $671 \mathrm{~nm}$ and trapped in a volume of approximately $1 \mathrm{~mm}$ diameter. In steady state, about $25 \%$ of the atoms in the trap are in the excited $2 p$ state. A fraction of about $93 \%$ of these atoms are in a single magnetic sublevel with $\left|m_{\ell}\right|=1$ with respect to the direction of a weak homogeneous magnetic field (about 5 Gauss), which is referred to as the $z$ direction below. As detailed in [24], this high degree of polarization is achieved by an appropriate choice of the frequency as well as the polarizations of the cooling laser beams exploiting the Zeeman splitting of the magnetic sublevels as well as the dipole selection rules, respectively. This way an optical-pumping scheme is employed resulting in an average increase of the magnetic quantum number for each absorbed photon.

The femtosecond laser is based on a Ti:Sa oscillator with two noncollinear optical parametric amplifier (NOPA) stages similar to the setup reported in [27]. The oscillator provides pulses with wavelengths ranging from $660 \mathrm{~nm}$ to about $1000 \mathrm{~nm}$ with a pulse duration of $5 \mathrm{fs}$, a repetition rate of $80 \mathrm{MHz}$, and an average power of $200 \mathrm{~mW}$. These pulses are stretched by passing through a combination of sapphire and fused silica windows to a duration in the order of about 1 ps. These strongly chirped pulses are superposed with a synchronized pump beam in two NOPA stages. The pump beam operates at a wavelength of $515 \mathrm{~nm}$ with a rate and duration of $200 \mathrm{kHz}$ and $250 \mathrm{fs}$, respectively. Changing the relative timing between the pump pulse and the chirped oscillator pulse allows us to control the part of the spectrum that is amplified in the NOPA stages. In this way, the center wavelength can be tuned over the full wavelength range

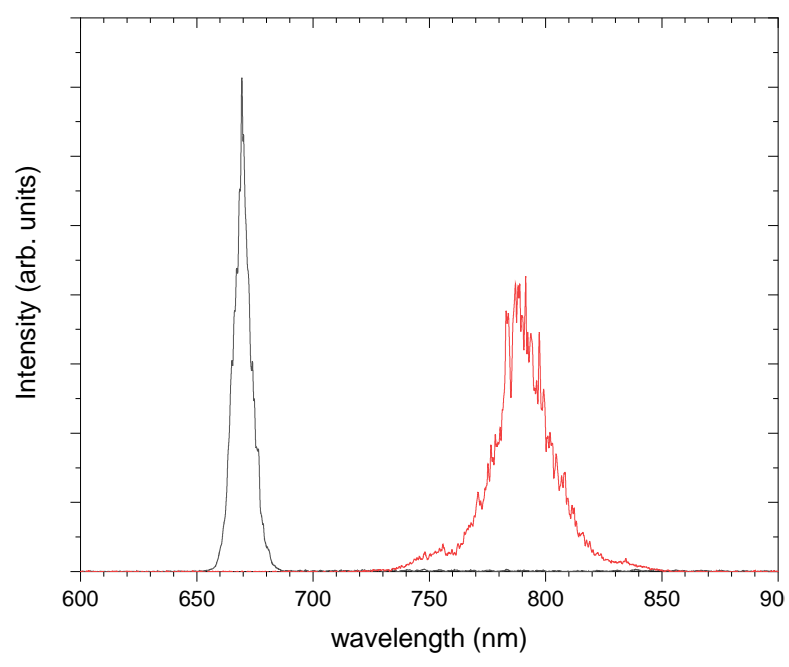

FIG. 1. Typical emission spectra of the femtosecond light source with center wavelengths tuned to $665 \mathrm{~nm}$ (black curve) and $795 \mathrm{~nm}$ (red curve).

of the oscillator. Behind the NOPA stages, the pulses are recompressed by a set of chirped mirrors to a pulse length of several tens of fs. The laser is guided through the vacuum chamber at a small angle $\left(10^{\circ}\right)$ with respect to the $z$ direction and focused on the target cloud with a beam waist of $50 \mu \mathrm{m}$. Peak laser intensities of up to $I=1.3 \times 10^{12} \mathrm{~W} / \mathrm{cm}^{2}$ were reached. Two typical spectra for center wavelengths of $670 \mathrm{~nm}$ and $790 \mathrm{~nm}$ are shown in Fig. 1.

The electron and ion momentum spectrometers are described in more detail in [25]. Briefly, electrons and target ions are extracted in opposite directions along the $z$ axis by weak homogeneous electric and magnetic fields and detected with position- and time-sensitive microchannel plate detectors. The particles' positions and time-of-flights allow us to determine their initial momenta [28, 29]. As mentioned above, the target cloud contains atoms in the $2 s$ ground state and the excited $2 p$ state. In order to separate the cross sections for the two initial states, the cooling lasers were switched off for $1 \mu \mathrm{s}$ during every other femtosecond laser pulse. On the one hand, this switch-off duration is much longer than the lifetime of the excited $2 p$ state $(27 \mathrm{~ns})$. Therefore, the spectra measured for the cooling lasers being switched off correspond to pure ground-state $(2 s)$ ionization. On the other hand, the switch-off duration is much shorter than typical timescales of the thermal motion $(v \sim 1 \mu \mathrm{m} / \mu \mathrm{s})$ of the atoms in the AOT. Therefore, the number density of the target atoms does not change significantly during the switching cycle, and the cross sections for the $2 p$ initial state can be calculated by subtracting the $2 s$ cross sections from the spectra measured for the cooling lasers being switched on by using an appropriate scaling factor that only depends on the previously determined fraction of atoms being in the excited state. Notably, knowing the excited population fraction allows also to cross-normalize 


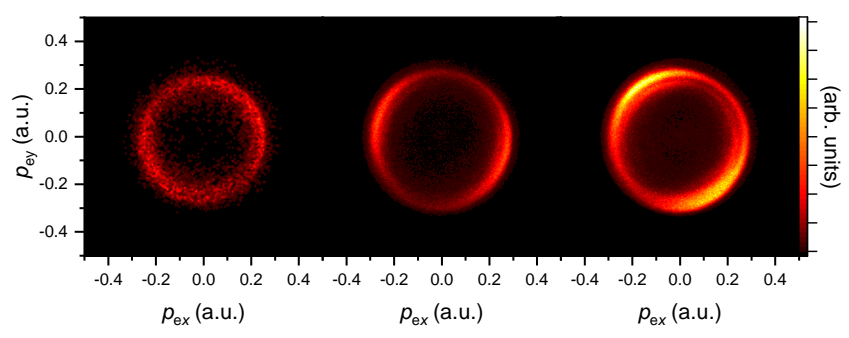

FIG. 2. Electron momentum distributions in the $x y$ plane for ionization of the $2 s$ (left), counter-rotating $2 p$ (center), and co-rotating $2 p$ state (right) at a center wavelength of $795 \mathrm{~nm}$ and a peak intensity of $0.8 \times 10^{12} \mathrm{~W} / \mathrm{cm}^{2}$. The color scale is linear, and the spectra are cross-normalized but multiplied by a factor of 100 for $2 s$ ionization.

the cross sections for the two initial states.

Typical electron momentum distributions integrated in the $z$ direction are shown in Fig. 2 for $2 s$ ionization and $2 p$ ionization with co- and counter-rotating helicities. In the present experiment, a resolution of 0.01 a.u. in the direction perpendicular to the femtosecond laser beam and of 0.005 a.u. in the direction longitudinal to it was achieved. For ionization with light of circular polarization in the $x y$ plane, the momentum distributions shown in the figure should feature circular symmetry. However, in the experiment an enhancement in two opposite directions is observed. This is caused by the imperfect polarization of both the target atoms as well as the laser radiation, which is estimated to have an ellipticity of about $20 \%$.

\section{THEORETICAL MODEL}

The experimental efforts are supported by $a b i n i$ tio calculations based on solving the time-dependent Schrödinger equation (TDSE) in the single-active electron (SAE) picture for a $(n \ell)$ active electron in a He-like $1 s^{2}$ ionic core. Details of the potential, which is based on the static Hartree potential [30,31] supplemented by phenomenological terms, are given in [19]. We achieved an accuracy of better than $1 \mathrm{meV}$ for the $n=2$ and $n=3$ ionization potentials (IPs). The initial state of the TDSE is propagated in the velocity gauge, and the ionization probability is computed by projecting the timedependent wavefunction at the end of the pulse onto scattering states. The earlier TDSE results exhibited excellent agreement with our experimental data for the photoelectron spectra and momenta measured under similar experimental conditions [19].

\section{RESULTS AND DISCUSSION}

For the laser wavelengths and intensities investigated in this study, the Keldysh parameter $\gamma$ for the valence ionization of lithium is always much larger than unity, such that the process can be described in the

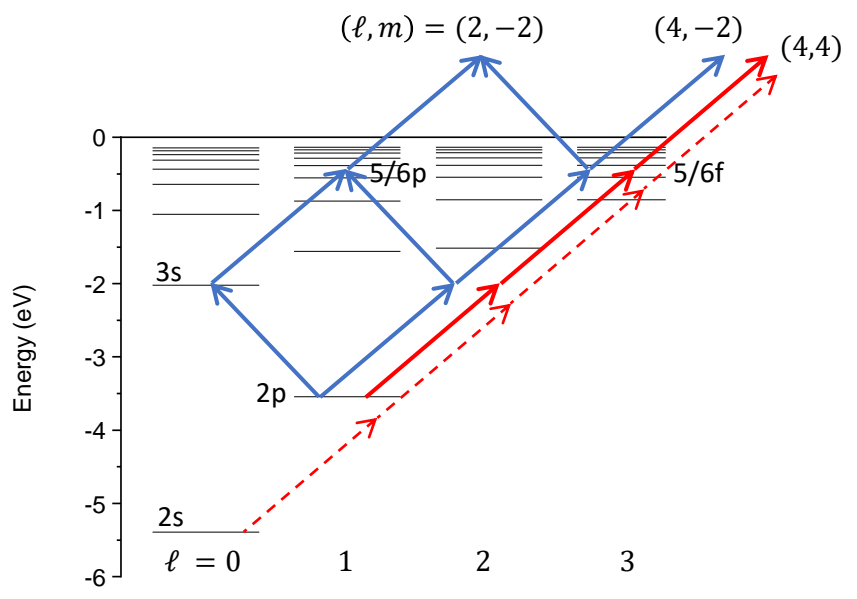

FIG. 3. Ionization scheme for three-photon ionization of the co- (solid red arrows) and counter-rotating (solid blue arrows) $2 p$ excited state as well as for four-photon ionization of the $2 s$ ground state (dashed red arrows).

multi-photon picture in lowest-order perturbation theory (LOPT) [32]. Here, the absorption of one photon of righthanded circular polarization propagating along the quantization direction (i.e. the $z$ direction) results in a change of the magnetic quantum number $\Delta m=+1$, while the absorption of a left-handed circularly polarized photon leads to $\Delta m=-1$. The resulting pathways for the threephoton ionization of $\mathrm{Li}(2 p, m=+1)$ by co- and counterrotating fields $(\lambda=795 \mathrm{~nm})$ are depicted in Fig. 3 as red and blue arrows, respectively. There is only a single possible pathway for co-rotating ionization with the electron being promoted to a final continuum state with $(\ell, m)=(4,4)$. For the counter-rotating case, in contrast, the final state is a superposition of $(\ell, m)=(2,-2)$ and $(4,-2)$ partial waves, respectively. Around a wavelength of $812 \mathrm{~nm}$ (neglecting the dressing of the states in the field), resonance enhancement is expected for counterrotating polarization due to the presence of the $3 s$ state. Two-photon resonances contribute, for either polarization, via the $n=5$ levels at about $826 \mathrm{~nm}-829 \mathrm{~nm}, n=6$ at about $780 \mathrm{~nm}, n=7$ at $760 \mathrm{~nm}, n=8$ at $744 \mathrm{~nm}$, etc.. The two-photon ionization threshold is reached at $700 \mathrm{~nm}$. For 4-photon ionization of $\operatorname{Li}(2 s)$ (dashed red arrows in Fig. 3), the final angular-momentum state is, respectively, $(\ell, m)=(4,-4)$ or $(4,4)$ for left or righthanded circular polarizations, resulting in identical cross sections for the two light helicities.

Different angular momenta contribute to the photoelectron angular distributions (PADs). The expected intensity distributions for the individual partial waves $(\ell, m)=(4,4),(2,-2)$, and $(4,-2)$ are shown in the top left panel of Fig. 4 as a function of the polar angle $\vartheta$. For $(\ell, m)=(4,4)$, the $\vartheta$ dependence is given by a $\sin ^{8}(\vartheta)$-function with a narrow peak perpendicular to the quantization axis. A similar behavior is observed for $(\ell, m)=(2,-2)$ with a slightly broader peak following a $\sin ^{4}(\vartheta)$-distribution. The $g$ state with $m=-2$, in con- 

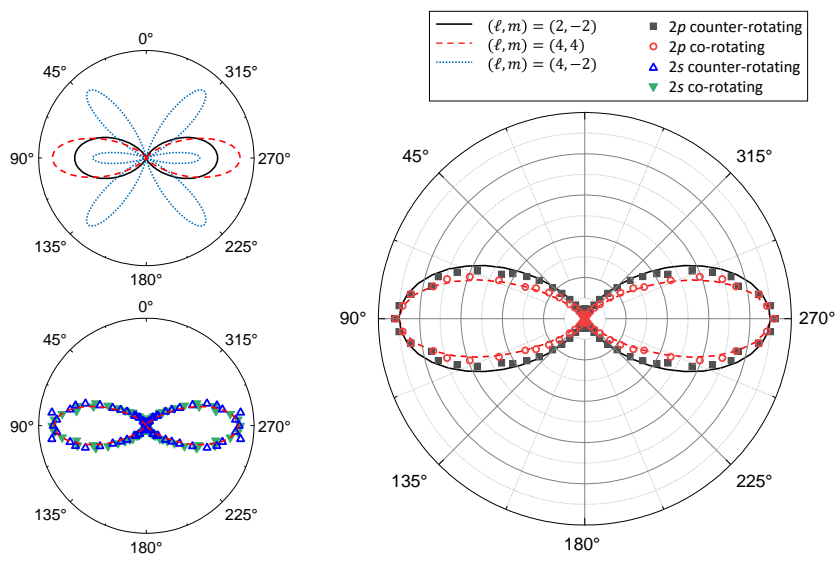

FIG. 4. Photoelectron angular distributions for ionization by a laser field with a center wavelength of $795 \mathrm{~nm}$ and a peak intensity of $0.8 \times 10^{12} \mathrm{~W} / \mathrm{cm}^{2}$. Top left: $\vartheta$ distribution of partial waves corresponding to the spherical harmonics $Y_{4}^{4}$ (dashed red), $Y_{4}^{-2}$ (dotted blue), and $Y_{2}^{-2}$ (solid black). Bottom left: Measured $\vartheta$ distribution for $2 s$ ionization for left(blue open triangles) and right-handed (green solid triangles) circularly polarized light along with the distribution of the partial wave with $(\ell, m)=(4,4)$ (dashed red line). Right: Measured $\vartheta$ distribution for $2 p$ ionization for co- (black solid squares) and counter-rotating (red open circles) cases with the partial waves $(\ell, m)=(4,4)$ (dashed red line) and $(2,-2)$ (solid black line).

trast, features intensity not only at $90^{\circ}$ but also at $40^{\circ}$ and $140^{\circ}$ with respect to the laser beam direction.

The comparisons to the experimental data for a center wavelength of $795 \mathrm{~nm}$ are shown in the two other panels of Fig. 4. As expected, the experimental electron angular distributions for $2 s$ ionization are very well described by the $\sin ^{8}$-distribution of a $g$ state (bottom left in the figure) for both helicities. The same behavior is observed for $2 p$ ionization in the co-rotating geometry (Fig. 4, right). For the counter-rotating scheme, the $\vartheta$ dependence matches well with the $\sin ^{4}$-distribution of the $d$ state. Notably, the cross section is rather small at angles where the $(\ell, m)=(4,-2)$ partial wave has its highest intensities, indicating that the final state is vastly dominated by the $\ell=2$ wave. Qualitatively, the dominance of this $d$ state can be explained by ionization enhancement through the $2 p-3 s$ resonance, because in LOPT this pathway necessarily results in final $\ell=2$ states. At other wavelengths, and in absence of the $2 p-3 s$ resonance, the angular distribution can be vastly different (see e.g. [19]).

Figure 5 depicts electron energy distributions for a laser wavelength of $795 \mathrm{~nm}$ with intensities of $0.8 \times 10^{12} \mathrm{~W} / \mathrm{cm}^{2}$ and $1.3 \times 10^{12} \mathrm{~W} / \mathrm{cm}^{2}$. For $2 s$ ionization, the spectra exhibit a single peak around $0.9 \mathrm{eV}$. For $2 p$ ionization, the peak is at slightly higher energies around $1.1 \mathrm{eV}$. These values agree with the expectations for four- and three-photon ionization of the two respective initial states. In our earlier experiment studying the ionization of the same atomic system at a shorter wave-

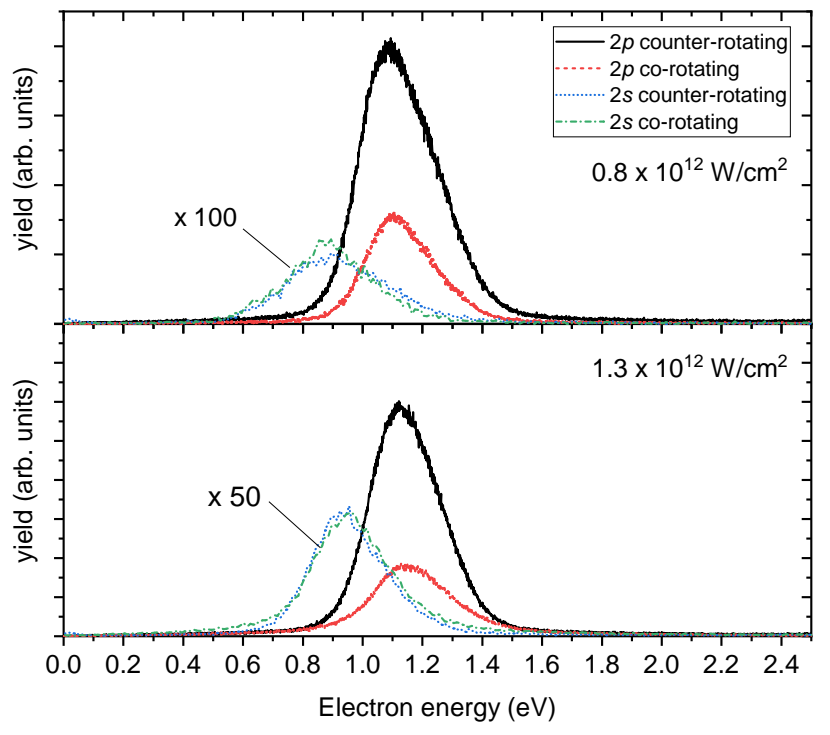

FIG. 5. Photoelectron energy distributions for $2 s$ and $2 p$ ionization by a laser field with a center wavelength of $795 \mathrm{~nm}$ and peak intensities of $0.8 \times 10^{12} \mathrm{~W} / \mathrm{cm}^{2}$ (top) and $1.3 \times 10^{12} \mathrm{~W} / \mathrm{cm}^{2}$ (bottom).

length close to the $2 s-2 p$ resonance [19], intensity- and helicity-dependent photoelectron energy shifts of up to $40 \%$ were observed and explained by the Autler-Townes effect, which can be very strong in the vicinity of resonances. In the present case, such energy shifts are substantially smaller due to the much larger detuning of the laser center wavelength with respect to the resonance ( $\Delta \lambda \approx 20 \mathrm{~nm}$ as compared to $5 \mathrm{~nm}$ in the previous study) and the lower spectral density of the radiation (cf. Fig. 1).

The energy spectra shown in Fig. 5 are crossnormalized for the two laser field helicities exploiting the fact that the ionization cross sections for the spherically symmetric $2 s$ initial state should be identical for both handednesses of the light. As can be seen from the figure, the ionization of the $2 p$ state is strongly favored for the counter-rotating geometry. Using Eq. (1), this helicity dependence can be quantified. Negative $C D$ values of about $-0.49 \pm 0.1$ and $-0.54 \pm 0.1$, respectively, are obtained for both intensities. The error provided here is an estimate considering the statistical error as well as the uncertainty in the excitation ratio of the atoms in the AOT, which is about $25 \pm 3 \%$. Additional systematic uncertainties arise due to the imperfect polarization of both the laser radiation and the target atoms, as well as small drifts in the wavelength and intensity of the laser pulses during the measurements.

The intensity dependence of the $C D$ values was investigated in a series of measurements between $0.5 \times 10^{12} \mathrm{~W} / \mathrm{cm}^{2}$ and $1.3 \times 10^{12} \mathrm{~W} / \mathrm{cm}^{2}$ at a wavelength of $\lambda=795 \mathrm{~nm}$. The obtained data are plotted in Fig. 6 (left) along with the values measured in our earlier experiment at $\lambda=665 \mathrm{~nm}$, which are (partly) published in $[19,33]$. For both wavelengths, the intensity dependence 

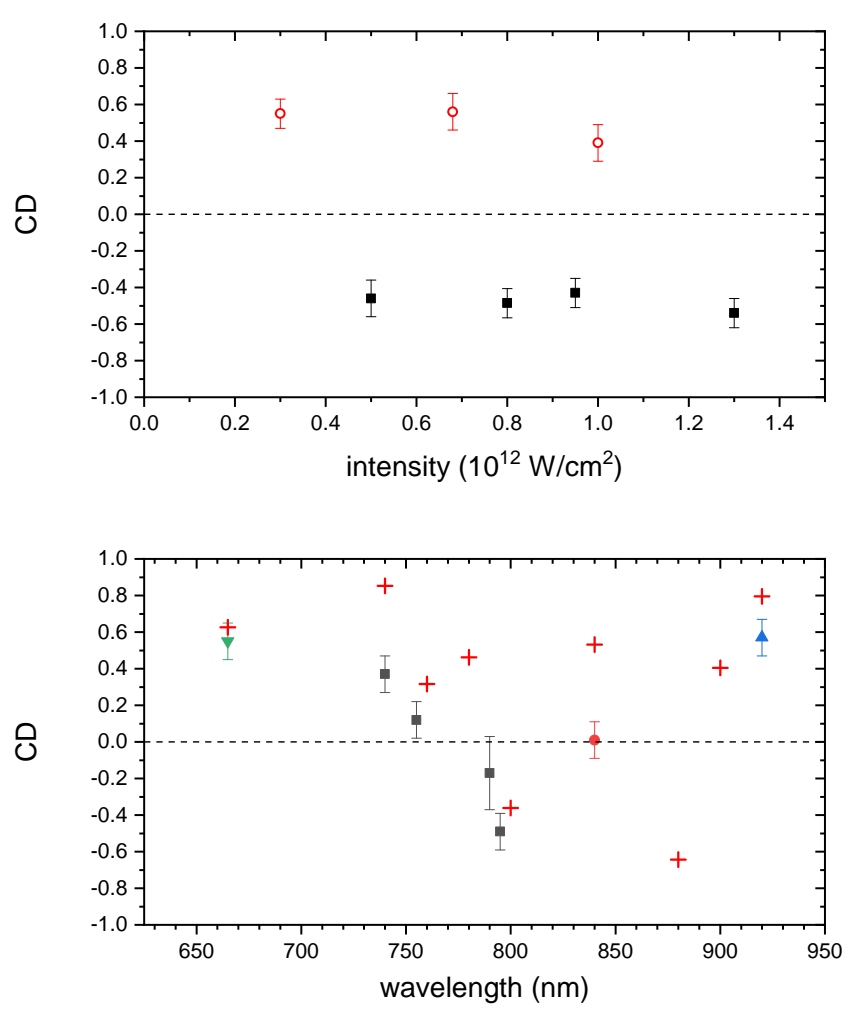

FIG. 6. Top: $C D$ values as a function of laser intensity for a center wavelength of $795 \mathrm{~nm}$ (black solid squares) and $665 \mathrm{~nm}$ (red open circles). Bottom: $C D$ values as a function of the center wavelength. The experimental data were measured for peak intensities of $0.3 \times 10^{12} \mathrm{~W} / \mathrm{cm}^{2}$ (green and blue triangles), $0.4 \times 10^{12} \mathrm{~W} / \mathrm{cm}^{2}$ (red circle), and $0.5 \times 10^{12} \mathrm{~W} / \mathrm{cm}^{2}$ (solid squares). The laser spectrum at $920 \mathrm{~nm}$ was substantially broader $( \pm 70 \mathrm{~nm})$ than in the other cases. The calculated $C D$ values (red crosses) are for $35 \mathrm{fs}$ (FWHM) Fourierlimited laser pulses with a peak intensity of $0.3 \times 10^{12} \mathrm{~W} / \mathrm{cm}^{2}$.

is fairly weak. It should be noted that it is not possible to measure the cross sections at a single intensity in our experiment due to the spatial extension of the reaction volume and the intensity distribution around the focal point of the laser beam. The intensities given in the graphs correspond to their highest values while the measured data represent averages over the intensity distribution in the focal volume [34]. Notably, the $C D$ values have opposite sign for the two wavelengths. As mentioned above, the difference can be explained by the $2 p-3 s$ resonance that enhances the ionization rate for the counter-rotating case around $815 \mathrm{~nm}$.

The above observation suggests that, in the intensity and wavelength regime considered, the dependence of the $C D$ on the wavelength of the incoming field is much stronger than its dependence on intensity. To obtain further insight, we varied the wavelength of the laser field between $740 \mathrm{~nm}$ and $795 \mathrm{~nm}$ at a constant intensity of about $5 \times 10^{11} \mathrm{~W} / \mathrm{cm}^{2}$ and measured the corresponding $C D$ values. In this region, the $C D$ monotonically drops from positive to negative values (see Fig. 6). When further measurements at higher and lower wavelengths with slightly different spectral widths and intensities were performed, a local minimum of the $C D$ was observed at the $2 p-3 s$ resonance.

The measured $C D$ values were compared with the results from the TDSE calculations. We used 35 fs FWHM (corresponding to a bandwidth of about $60 \mathrm{meV}$ ) Fourierlimited pulses with Gaussian envelopes and a peak intensity of $0.3 \times 10^{12} \mathrm{~W} / \mathrm{cm}^{2}$. We observe an overall good agreement between the measurements and the calculations (see Fig. 6). Note that the negative $C D$ value around $800 \mathrm{~nm}$ is well reproduced theoretically. The calculation even predicts a second minimum with a negative $C D$ value near $880 \mathrm{~nm}$. However, no experimental data are available at this wavelength.

To obtain a better understanding of the alternating behavior of the $C D$ values between $800 \mathrm{~nm}$ and $880 \mathrm{~nm}$, we monitored the population of the (undressed) bound states (with $n \leq 8$ ) in time. Even though it is not possible to unambiguously trace the population transfer from one specific state to another, this method still enables us to identify resonances that are relevant for ionization enhancement.

For the co-rotating field, the time-dependent populations are shown in Fig. 7 for $800 \mathrm{~nm}, 840 \mathrm{~nm}$, and $880 \mathrm{~nm}$ light. The dressing of the initial $2 p$ state by the weak laser field occurs mainly via one-photon coupling to the $2 s$ and $3 d$ states, whose populations are seen to adiabatically follow the square of the pulse envelope, leading to the well-known quadratic AC stark shift. It is noteworthy that these states mix strongly, although there is a large frequency detuning to the $2 s-2 p$ and $2 p-3 d$ transitions at $671 \mathrm{~nm}$ and $610 \mathrm{~nm}$, respectively. On the other hand, resonant transitions to the excited $5 f$ and $6 f$ Rydberg states participate very effectively in the ionization enhancement. These states, coupled to the $2 p$ state by two-photon transitions, undergo Rabi-like oscillations [35-37], whose frequency depends on the strength of the coupling, leading to nonnegligible populations at the end of the pulse. The ionization enhancement is strongest at $840 \mathrm{~nm}$ due to the coupling between the $2 p$ and $5 f$ states, whose resonance wavelength is $826 \mathrm{~nm}$. In this case, the total ionization probability is more than five times higher than for the other two wavelengths, and a significant fraction of the population (more than $5 \%$ ) remains in the excited $5 f$ state after the pulse. At $880 \mathrm{~nm}$, the $4 f$ state is seen to mostly contribute to a quartic AC stark shift via two-photon coupling.

The population distributions for the counter-rotating case are shown in Fig. 8. As expected, prominent Rabi oscillations and a large population transfer (up to $90 \%$ ) between the initial $2 p$ and the excited $3 s$ state appear at $800 \mathrm{~nm}$ due to the proximity to the resonance. From the $3 s$ state, population can be transferred further up to the continuum using the $6 p$ state as a stepping stone (see Fig. 3), resulting in an ionization probability well above $1 \%$. At $840 \mathrm{~nm}$, the overall picture remains similar with 


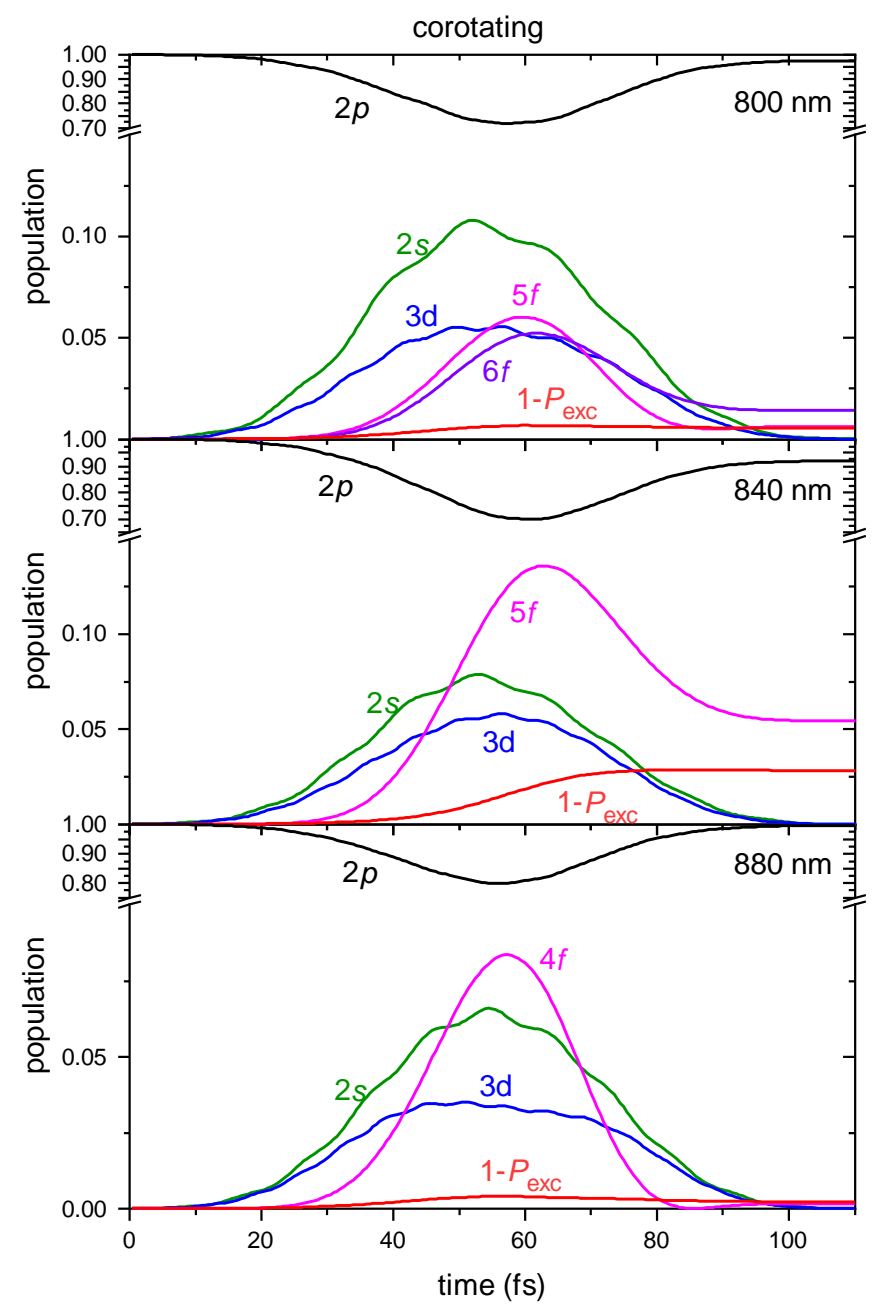

FIG. 7. Theoretical time-dependent atomic populations during a co-rotating pulse with peak intensities of $0.3 \times 10^{12} \mathrm{~W} / \mathrm{cm}^{2}$ and center wavelengths of $800 \mathrm{~nm}$ (top), $840 \mathrm{~nm}$ (middle), and $880 \mathrm{~nm}$ (bottom). The line denoted with $\left(1-P_{\text {exc }}\right)$ mainly corresponds to the population of the continuum states, although it contains a small contribution from states with $n>8$.

a weaker coupling between the $2 p$ and $3 s$ states leading to a slightly reduced ionization probability. Even further off resonance at $880 \mathrm{~nm}$, the amplitudes of the Rabi oscillations are strongly reduced and the 3 s population follows the pulse envelope more adiabatically. It is surprising that the ionization rate increases again for this wavelength. However, there are two features of the population distributions that might explain the overall high ionization rate: First, the $3 s$ population is consistently above $10 \%$ during the pulse and even approaches $20 \%$ when the field intensity reaches its maximum. Second, two-photon excitation of the $4 f$ state is observed as well, which might contribute to the large ionization probability at this wavelength. Overall, the variation of the ionization probability is much smaller for the counter-rotating geometry than for the co-rotating one. Therefore, we

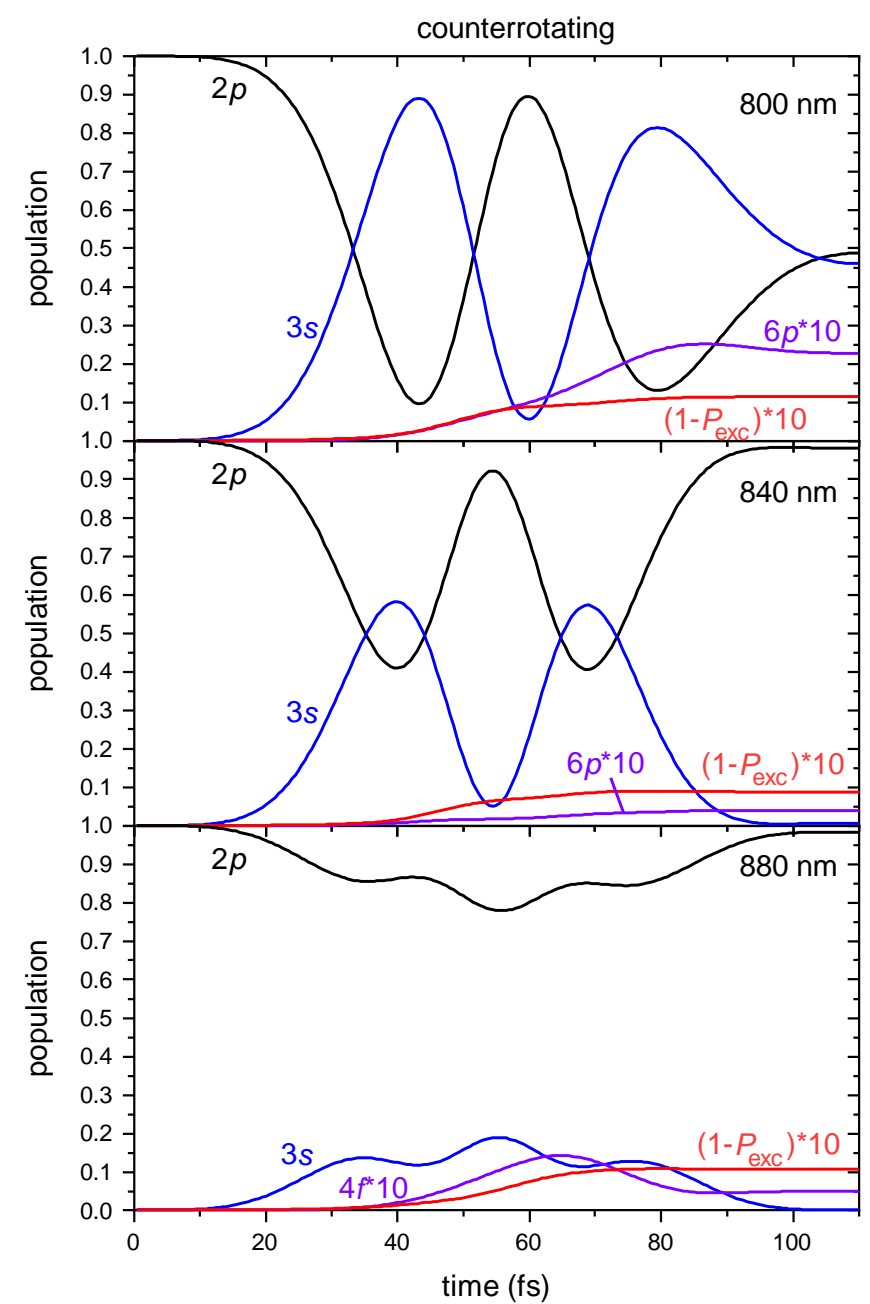

FIG. 8. Same as Fig. 7, but for counter-rotating geometry.

attribute the alternating behavior of the $C D$ mainly to the large fluctuations of the ionization probability for the co-rotating field.

This theoretical analysis shows that the ionization rate depends strongly on the coherent population transfer between the initial and intermediate states. Therefore, it is in principle possible to induce sharp variations of the $C D$ through only small variations of the wavelength. Because the populations of the states undergo Rabi oscillations, the $C D$ is also affected by the intensity, duration, and wavelength of the pulse. Since the cross sections are not measured for a single peak intensity but for an intensity range spanning the laser focus, along with other experimental uncertainties, our measured cross sections and $C D$ values represent an average that results in a smoother variation of the $C D$ than in theory. These effects make the identification of intensity-related variations almost impossible, or at least very challenging, from an experimental perspective. Due to the remaining uncertainties and fluctuations in several of the experimental parameters, we did not perform additional calcu- 
lations as they would most likely not shed more light on the interpretation of the experimental data.

It should be noted that there are also significant discrepancies between the measured and calculated $C D$ values at wavelengths of $740 \mathrm{~nm}, 780 \mathrm{~nm}$, and $840 \mathrm{~nm}$. All these wavelengths are close to two-photon resonances between the $2 p$ and excited $n p$ and $n f$ states, which lie around $826-829 \mathrm{~nm}$ for $n=5,780 \mathrm{~nm}$ for $n=6$, and $744 \mathrm{~nm}$ for $n=8$. Although we did not perform a detailed investigation of the population distributions for these wavelengths, we expect that two-photon Rabi oscillations with periods close to the pulse duration are also strongly affecting the efficiency of resonance enhancement.

\section{SUMMARY AND CONCLUSIONS}

In this work, we addressed the question how atomic resonances affect the helicity dependence of the multiphoton ionization of polarized atomic targets in circularly polarized femtosecond laser fields. Specifically, we studied circular dichroism in two- and three-photon ionization of excited and polarized lithium atoms initially in the $2 p\left(m_{\ell}=+1\right)$ state for wavelengths ranging from $665 \mathrm{~nm}$ to $920 \mathrm{~nm}$. Experimentally, we find that the ionization probability is larger for most wavelengths if the electric field vector and the initial electronic current density are co-rotating. However, there is a distinct minimum in the $C D$ value at a wavelength near $800 \mathrm{~nm}$, where the counter-rotating geometry is favored. This feature is qualitatively explained by the $2 p-3 s$ resonance, which is only driven for the counter-rotating case, thus resulting in a helicity-selective resonance enhancement.

The comparison of the experimental data to our theoretical model, which is based on the numerical solution of the time-dependent Schrödinger equation, yields good qualitative agreement, although the calculation shows a larger scattering of the $C D$ values and even a second minimum around $880 \mathrm{~nm}$. The theoretical approach allows to extract time-dependent population distributions of the atomic states during and after the femtosecond laser pulse, which helps to shed light on the underlying dynamics and the involved intermediate states. This analysis shows Rabi oscillations between states coupled by one- and two-photon transitions. The populated excited states can result in resonance-enhancement and act as stepping stones for the electrons while they are promoted to the continuum. Because Rabi frequencies and time-dependent excited state populations depend sensitively on the details of the pulse, such as its intensity, duration, and detuning off a resonance, the total ionization probabilities and, therefore, the $C D$ values are expected to be strongly influenced by these parameters as well. In our experiment, the peak intensity of the laser pulse is difficult to control due to the spatial intensity profile of the laser focus in the reaction region. As a result, the experimental $C D$ values represent a weighted average for different intensities.

The present work does not only show the importance of the atomic structure on the ionization probability and the circular dichroism, but it also exposes the significance of details of the pulse parameters on the ionization dynamics.

\section{ACKNOWLEDGMENTS}

The experimental material presented here is based upon work supported by the National Science Foundation under Grant No. PHY-1554776. The theoretical part of this work was funded by the NSF under grants No. PHY-2012078 (T.M. and N.D.) and PHY-1803844 (K.B.), and by the XSEDE supercomputer allocation No. PHY-090031. The calculations were carried out on Comet at the San Diego Supercomputer Center and Bridges-2 (via a trial allocation) at the Pittsburgh Supercomputing Center.
[1] N. Berova, P. L. Polavarapu, K. Nakanishi, and R. W. Woody, eds., Comprehensive Chiroptical Spectroscopy (Wiley, 2014).

[2] N. Böwering, T. Lischke, B. Schmidtke, N. Müller, T. Khalil, and U. Heinzmann, Phys. Rev. Lett. 86, 1187 (2001).

[3] G. A. Garcia, L. Nahon, S. Daly, and I. Powis, Nature Communications 4, 2132 (2013).

[4] L. Nahon, G. A. Garcia, and I. Powis, Journal of Electron Spectroscopy and Related Phenomena 204, 322 (2015), gas phase spectroscopic and dynamical studies at Free-Electron Lasers and other short wavelength sources.

[5] R. E. Goetz, C. P. Koch, and L. Greenman, Phys. Rev. Lett. 122, 013204 (2019).

[6] C. Lux, M. Wollenhaupt, T. Bolze, Q. Liang, J. Köhler, C. Sarpe, and T. Baumert, Angewandte Chemie Inter- national Edition 51, 5001 (2012).

[7] C. Lux, A. Senftleben, C. Sarpe, M. Wollenhaupt, and T. Baumert, Journal of Physics B: Atomic, Molecular and Optical Physics 49, 02LT01 (2015).

[8] S. Beaulieu, A. Comby, D. Descamps, B. Fabre, G. A. Garcia, R. Géneaux, A. G. Harvey, F. Légaré, Z. Mašín, L. Nahon, A. F. Ordonez, S. Petit, B. Pons, Y. Mairesse, O. Smirnova, and V. Blanchet, Nature Physics 14, 484 (2018).

[9] A. Jorissen and C. Cerf, Origins of life and evolution of the biosphere 32, 129 (2002).

[10] M. Tia, B. C. de Miranda, S. Daly, F. Gaie-Levrel, G. A. Garcia, I. Powis, and L. Nahon, The Journal of Physical Chemistry Letters 4, 2698 (2013).

[11] M. Tia et al. The Journal of Physical Chemistry Letters 8, 2780 (2017). 
[12] T. Herath, L. Yan, S. K. Lee, and W. Li, Phys. Rev. Lett. 109, 043004 (2012).

[13] C. A. Mancuso, K. M. Dorney, D. D. Hickstein, J. L. Chaloupka, X.-M. Tong, J. L. Ellis, H. C. Kapteyn, and M. M. Murnane, Physical Review A 96, 023402 (2017).

[14] S. Eckart et al., Nature Physics 14, 701 (2018).

[15] U. S. Sainadh et al., Nature 568, 75 (2019).

[16] T. Mazza et al., Nature Communications 5, 3648 (2014).

[17] M. Ilchen et al., Phys. Rev. Lett. 118, 013002 (2017).

[18] A. N. Grum-Grzhimailo, N. Douguet, M. Meyer, and K. Bartschat, Phys. Rev. A 100, 033404 (2019).

[19] A. H. N. C. de Silva, D. Atri-Schuller, S. Dubey, B. Acharya, K. Romans, K. Foster, O. Russ, K. Compton, C. Rischbieter, N. Douguet, K. Bartschat, and D. Fischer, Phys. Rev. Lett. 126, 023201 (2021).

[20] H. A. Bethe and E. E. Salpeter, Quantum mechanics of one and two electron atoms (Plenum, New York, 1977).

[21] I. Barth and O. Smirnova, Phys. Rev. A 84, 063415 (2011).

[22] J. H. Bauer, F. Mota-Furtado, P. F. O'Mahony, B. Piraux, and K. Warda, Phys. Rev. A 90, 063402 (2014).

[23] R. R. Freeman, P. H. Bucksbaum, H. Milchberg, S. Darack, D. Schumacher, and M. E. Geusic, Phys. Rev. Lett. 59, 1092 (1987).

[24] S. Sharma, B. P. Acharya, A. H. N. C. de Silva, N. W. Parris, B. J. Ramsey, K. L. Romans, A. Dorn, V. L. B. de Jesus, and D. Fischer, Phys. Rev. A 97, 043427 (2018).

[25] R. Hubele et al., Review of Scientific Instruments 86, 033105 (2015).

[26] F. Thini et al., J. Phys. B 53, 095201 (2020).
[27] A. Harth, C. Guo, Y.-C. Cheng, A. Losquin, M. Miranda, S. Mikaelsson, C. M. Heyl, O. Prochnow, J. Ahrens, U. Morgner, A. L'Huillier, and C. L. Arnold, Journal of Optics 20, 014007 (2017).

[28] J. Ullrich, R. Moshammer, A. Dorn, R. Dörner, L. Ph. H. Schmidt, and H. Schmidt-Böcking, Rep. Prog. Phys. 66, 1463 (2003).

[29] D. Fischer, in Ion-Atom Collisions, edited by M. Schulz (De Gruyter, 2019) pp. 103-156.

[30] B. J. Albright, K. Bartschat, and P. R. Flicek, Journal of Physics B: Atomic, Molecular and Optical Physics 26, 337 (1993).

[31] M. Schuricke, G. Zhu, J. Steinmann, K. Simeonidis, I. Ivanov, A. Kheifets, A. N. Grum-Grzhimailo, K. Bartschat, A. Dorn, and J. Ullrich, Phys. Rev. A 83, 023413 (2011).

[32] P. Lambropoulos, in Advances in Atomic and Molecular Physics Volume 12 (Elsevier, 1976) pp. 87-164.

[33] A. H. N. C. de Silva, Symmetry-breaking in the multiphoton ionization dynamics of oriented atoms, Ph.D. thesis, Missouri University of Science and Technology (2020).

[34] M. Schuricke, G. Zhu, J. Steinmann, K. Simeonidis, I. Ivanov, A. Kheifets, A. N. Grum-Grzhimailo, K. Bartschat, A. Dorn, and J. Ullrich, Phys. Rev. A 83, 023413 (2011).

[35] T. R. Gentile, B. J. Hughey, D. Kleppner, and T. W. Ducas, Phys. Rev. A 40, 5103 (1989).

[36] A. F. Linskens, I. Holleman, N. Dam, and J. Reuss, Phys. Rev. A 54, 4854 (1996).

[37] M. Fushitani, C.-N. Liu, A. Matsuda, T. Endo, Y. Toida, M. Nagasono, T. Togashi, M. Yabashi, T. Ishikawa, Y. Hikosaka, T. Morishita, and A. Hishikawa, Nature Photonics 10, 102 (2015). 